\title{
Solicitud de inicio de procedimiento de la República de Chile contra el Estado Plurinacional de Bolivia ante la Corte Internacional de Justicia por el aprovechamiento de las aguas del Río Silala (6 de junio del 2016)
}

Application starting proceedings by the Republic of Chile against the Plurinational State of Bolivia with regard to a dispute concerning the status and use of the waters of the Silala River June $6^{\text {th }}$, 2016)

Al Sr. Secretario de la Corte Internacional de Justicia,

1. Yo, quien suscribe, debidamente autorizada por la República de Chile (en adelante "Chile"), país del cual soy Agente, tengo el honor de presentar a la Corte Internacional de Justicia, conforme al Artículo 36 y al Artículo 40 del Estatuto de la Corte, y al Artículo 38 de las Reglas de la Corte, una Solicitud de inicio de procedimiento a nombre de Chile contra el Estado Plurinacional de Bolivia (en adelante "Bolivia") en el siguiente asunto.

\section{Objeto de la controversia}

2. El sistema del Río Silala (también denominado Siloli) es un curso de agua internacional cuyas aguas superficiales nacen a unos 4.400 metros sobre el nivel del mar en territorio boliviano. En pocos kilómetros, fluye por tierra y cruza la frontera adentrándose en territorio chileno. El caudal superficial del Río Silala emana de vertientes de aguas subterráneas en las Quebradas de Orientales y Cajones, que se alimentan de un acuífero que se extiende por la frontera entre Bolivia y Chile. Aun en territorio boliviano, dichas aguas fluyen hacia un curso de agua común, el Río Silala, que escurre hacia Chile debido a la pendiente natural del terreno en una dirección sur-oeste 
3. La controversia entre la República de Chile y el Estado Plurinacional de Bolivia concierne la alegación de Bolivia que el sistema del Río Silala no es un curso de agua transfronterizo y, por tanto, Bolivia tiene derecho a usar el 100\% de sus aguas. Nunca se cuestionó el carácter de curso internacional del Río Silala, hasta que Bolivia por primera vez en 1999, reclamó sus aguas como exclusivamente bolivianas.

4. Según lo detallado en la presente Solicitud, Chile pide que la Corte juzgue y declare que el sistema de aguas del Silala es de hecho y de derecho un curso de agua internacional cuyo uso por Chile y Bolivia se rige por el derecho internacional consuetudinario. Las precisas declaraciones que se piden son las indicadas en la Sección V contenida más adelante.

\section{Jurisdicción de la Corte}

5. La Corte tiene jurisdicción sobre la presente controversia de conformidad con lo dispuesto en el Artículo 36 de su Estatuto, en virtud de la aplicación del Artículo XXXI del Tratado Americano de Soluciones Pacíficas, el "Pacto de Bogotá", de 30 de abril de $1948^{1}$, que dice lo siguiente:

"De conformidad con el inciso $2^{\circ}$ del artículo 36 del Estatuto de la Corte Internacional de Justicia, las Altas Partes Contratantes declaran que reconocen con respecto a cualquier otro Estado Americano como obligatoria ipso facto, sin necesidad de ningún convenio especial mientras esté vigente el presente Tratado, la jurisdicción de la expresada Corte en todas las controversias de orden jurídico que surjan entre ellos y que versen sobre:

(a) La interpretación de un tratado;

(b) Cualquier cuestión de Derecho Internacional;

(c) La existencia de todo hecho que, si fuere establecido, constituiría violación de una obligación internacional;

(d) La naturaleza o extensión de la reparación que ha de hacerse por el quebrantamiento de una obligación internacional."

6. Tanto Bolivia como Chile son Partes en el Pacto de Bogotá. Chile ratificó el Pacto de Bogotá el 21 de agosto de $1967^{2}$. Bolivia lo hizo el 14 de abril de 2011, con reserva al Artículo VI en el sentido que "los procedimientos pacíficos pueden también aplicarse a

Tratado Americano de Soluciones Pacificas ("Pacto de Bogotá"), 30 de abril de 1948, UNTS Tomo Número 30, disponible en: https://treaties.un.org/doc/Publication/UNTS/Volume\%2030/volume-30-I-449-Other.pdf (Anexo 1).

2 República de Chile, Decreto $N^{\circ}$ 526, 21 de agosto de 1967, publicado el 6 de septiembre de 1967, disponible en: https://www.leychile.cl/Navegar?idNorma=400563 (Anexo 2). 
las controversias emergentes de asuntos resueltos por arreglo de Partes, cuando dicho arreglo afecta intereses vitales de un Estado" ${ }^{3}$. Bolivia retiró dicha reserva el 10 de abril de $2013^{4}$. Ninguna reserva pertinente que haya hecho alguna de las Partes se encuentra vigente en la actualidad.

7. Chile siempre ha estado dispuesto a entablar conversaciones con Bolivia respecto a un régimen de utilización de las aguas del Silala. Chile y Bolivia han sostenido tales conversaciones mediante una serie de reuniones bilaterales entre 2004 y 2010. A la larga, dichas conversaciones terminaron sin resultado alguno, debido a que Bolivia insistía en negar que el Río Silala es un curso de agua internacional y a que Bolivia alega que tiene derechos a usar el $100 \%$ de sus aguas.

8. El 27 de marzo de 2014, Chile envió una nota diplomática, reiterando su continuo interés y disposición para proceder con proyectos técnicos y colaboración mutua en el sistema del Río Silala ${ }^{5}$. En su respuesta, Bolivia respondió insistiendo una vez más en sus derechos soberanos exclusivos sobre este recurso hídrico compartido ${ }^{6}$.

9. El 23 de marzo de 2016, el Presidente de Bolivia Evo Morales afirmó (nuevamente) que el Río Silala no es un curso de agua internacional y anunció su intención de presentar una demanda internacional contra Chile por el uso ilegal de las aguas del Silala ${ }^{7}$. En estas circunstancias, Chile ha optado por entablar la actual demanda para resolver el asunto del estatus del Río Silala.

\section{Declaración de hechos}

10. El Río Silala nace de vertientes de aguas subterráneas en las Quebradas de Orientales y Cajones, ubicadas a unos 4.400 metros de altitud en Bolivia y a unos pocos kilómetros al

3 Estado Plurinacional de Bolivia, Nota OEA-SG-111-11, 9 de junio de 2011, adjuntando el Instrumento de Ratificación del "Pacto de Bogotá", con fecha 14 de abril de 2011 (Anexo 3.1). Se depositó el Instrumento de Ratificación ante el Secretario General de la OEA el 9 de junio de 2011. El 10 de junio de 2011, Chile presentó una objeción a la reserva de Bolivia y declaró que impedía la entrada en vigor del Pacto de Bogotá entre la República de Chile y el Estado Plurinacional de Bolivia, disponible en: http://www.oas.org/dil/esp/a-42_objecion_chile_06-15-2011.pdf (Anexo 3.2).

4 Estado Plurinacional de Bolivia, Nota MPB-OEA-ND-039-13, 8 de abril de 2013, adjuntando el Instrumento Retiro de Reserva al "Pacto de Bogotá", recibido el 10 de abril de 2013, disponible en: http://www.oas.org/dil/esp/Nota\%20de\%20Bolivia.\%20ND-039-13.pdf (Anexo 3.3).

5 Nota $N^{\circ}$ 96/72 del Ministerio de Relaciones Exteriores de Chile al Ministerio de Relaciones Exteriores del Estado Plurinacional de Bolivia, 27 de marzo de 2014 (Anexo 4).

6 Nota N ${ }^{\circ}$ VRE-DGLFAIT-UAIT-Cs-136/2014 del Ministerio de Relaciones Exteriores del Estado Plurinacional de Bolivia al Consulado General de Chile en Bolivia, 10 de abril de 2014 (Anexo 5).

7 Prensa Palacio, "Presidente Morales instruye estudiar alternativas jurídicas para defender aguas del Silala", La Paz - Bolivia, 23 de marzo de 2016, disponible en: http://www.presidencia.gob.bo/fuente/noticia.php?cod=4185 (Anexo 6.1). Véase también La Nación, "Evo Morales anuncia que acudirá a instancias internacionales por aguas del Silala", La Paz - Bolivia, 23 de marzo de 2016, disponible en: http://www.lanacion.cl/noticias/mundo/bolivia/evo-morales-anuncia-que-acudira-a-instanciasinternacionales-por-aguas/2016-03-23/113759.html (Anexo (6.2). 
noreste de la frontera internacional de Chile-Bolivia. El Río Silala cruza el límite internacional de Chile-Bolivia en un punto a unos 4 kilómetros al sureste del Cerro Inacaliri, en las coordenadas $22^{\circ} 00^{\prime} 34^{\prime \prime}$ S-68 $01^{\prime} 37^{\prime \prime W}$ (PSAD56) y a aproximadamente 4.278 metros de altitud. Al ingresar en territorio chileno, el caudal del Río Silala es de unos 160 l/s. En territorio chileno, el río recibe aguas adicionales de varias vertientes, incluyendo las de las Quebradas de Inacaliri y Negra, antes de llegar al Río Inacaliri. El Río Silala tiene un largo total de unos 8,5 kilómetros. De ese largo, unos 3,8 kilómetros están en territorio boliviano y 4,7 kilómetros están en territorio chileno.

11. Históricamente y por más de un siglo, las aguas del Río Silala han sido usadas en Chile para distintos propósitos, incluyendo el abastecimiento del suministro de agua a la ciudad de Antofagasta y a los pueblos de Sierra Gorda y Baquedano. Las aguas han sido además usadas con fines industriales por The Antofagasta (Chili) and Bolivia Railway Company Ltd. (también conocida como Ferrocarril de Antofagasta a Bolivia, en adelante "FCAB") y por diversas compañías mineras incluyendo la empresa estatal Corporación Nacional del Cobre ("CODELCO”).

12. Una antigua representación cartográfica del Río Silala aparece en el Mapa de las Cordilleras del 1884, hecho por Alejandro Bertrand y que fue encargado por Chile ${ }^{8}$. Muestra el "Río Cajón” (nombre usado en ese entonces para el Río Silala) en territorio boliviano, ingresando al territorio administrado por Chile según lo establecido bajo el Pacto de Tregua de 1884 entre las Partes, y confluyendo con el "Río S. Pedro" (la continuación del Río Inacaliri).

13. El Mapa Geográfico y Corográfico de la República de Bolivia del año 1890, por Justo Leigue Moreno, Sargento Mayor de la República de Bolivia, también muestra un curso de agua identificado como "Cajón" en territorio boliviano, confluyendo con el "Río S. Pedro" en territorio administrado por Chile, de nuevo según lo establecido bajo el Pacto de Tregua de $1884^{9}$.

14. Ambos Estados han reconocido al Río Silala como un curso de agua internacional que fluye de manera natural desde Bolivia hacia el interior de Chile en numerosos documentos. Estos incluyen el Tratado de Paz y Amistad de 1904 y documentos relacionados, y concesiones de derechos de agua concedidos por los dos gobiernos a FCAB en 1906 (Chile) y en 1908 (Bolivia).

15. El 20 de octubre de 1904, Chile y Bolivia suscribieron el Tratado de Paz y Amistad (mencionado arriba) que estableció el límite internacional definitivo entre ambos Estados $^{10}$. En dicha ocasión, los Estados firmantes aprobaron un mapa, también

Mapa de las Cordilleras por Alejandro Bertrand, 1884 (Anexo 7). Mapa Geográfico y Corográfico por Justo Leigue Moreno, 1890 (Anexo 8).

10 Tratado de Paz y Amistad celebrado entre Bolivia y Chile, 20 de octubre de 1904, publicado en el Diario Oficial N 8169 del 27 de marzo de 1905 (Anexo 9.1). 
firmado el 20 de octubre de 1904, por el Ministro de Relaciones Exteriores de Chile, Sr. Emilio Bello Codesido, y el Embajador de Bolivia en Chile, Sr. Alberto Gutiérrez. Este mapa representa en 1904 el "Río Silala" cruzando el límite entre Bolivia and Chile, entre el punto 15 (Cerro Silala) y el punto 16 (Cerro Inacaliri) de ese límite ${ }^{11}$.

16. El 23 de marzo de 1906, Julio Knaudt y Luis Riso Patrón, los Directores de Bolivia y Chile de las respectivas Comisiones de Límites, definieron la ubicación de las pirámides de hierro que demarcarían el límite, incluyendo una ubicada "En el río Silala"12. La existencia del Río Silala fue igualmente confirmada por la Comisión de Límites de Bolivia presidida por el ingeniero boliviano Quintín Aramayo Ortíz, durante la expedición de demarcación realizada entre el 28 de mayo y el 28 de julio de $1906^{13}$.

17. El 31 de julio de 1906, Chile otorgó una concesión a FCAB relacionada con el uso de las aguas del Río Silala en territorio chileno, por tiempo indefinido y con el fin de aumentar el caudal de agua que surtía al puerto de Antofagasta ${ }^{14}$.

18. El 28 de octubre de 1908, Bolivia también otorgó una concesión a FCAB, respecto a las aguas del Río Silala en territorio boliviano ${ }^{15}$. FCAB había solicitado el uso de las aguas para la operación de sus locomotoras de vapor y solicitado permiso para hacer obras de captación y de canalización en territorio boliviano ${ }^{16}$.

19. En 1942, la Comisión Mixta de Límites Chile-Bolivia (en adelante "Comisión Mixta de Límites") fue constituida conforme al Protocolo sobre Conservación de Hitos Fronterizos" ${ }^{17}$. La Comisión Mixta de Límites ha confirmado en múltiples ocasiones la existencia del Río Silala a ambos lados del límite.

20. El 7 de mayo de 1996, Bolivia emitió un Comunicado de Prensa oficial, respondiendo a ciertas aseveraciones publicadas en la prensa boliviana referidas a una desviación artificial de aguas del Río Silala hacia Chile. En el Comunicado de Prensa, Bolivia rechazó tales aseveraciones y confirmó que el Silala es un río que se origina en territorio boliviano e ingresa a territorio chileno, siendo Bolivia el país ribereño aguas arriba y Chile el país ribereño aguas abajo. Sin embargo, Bolivia dio credibilidad a la afirmación que las aguas

11 Mapa adjuntado al Tratado de Paz y Amistad, 20 de octubre de 1904 (Anexo 9.2).

12 Actas firmadas por Julio Knaudt y Luis Riso Patrón, 23 de marzo de 1906, en: Antecedentes Limites Chile-Bolivia, p. 2 (Anexo 10.1).

13 nforme firmado por Quintín Aramayo Ortíz, 14 de agosto de 1906, en: Antecedentes Limites Chile-Bolivia, pp. 14-18 (Anexo 10.2).

14 Escritura de Concesión por el Estado de Chile de las aguas del Siloli ( $\mathrm{N}^{\circ}$ 1892) a The Antofagasta (Chili) and Bolivia Railway Company Limited, 31 de julio de 1906 (Anexo 11).

15 Escritura de Concesión boliviana de las aguas del Siloli $\left(\mathrm{N}^{\circ} 48\right)$ a The Antofagasta (Chili) and Bolivia Railway Company Limited, 28 de octubre de 1908 (Anexo 12).

16 Ibid., p. 2.

17 Protocolo sobre Conservación de hitos Fronterizos, 10 de agosto de 1942 (Anexo 13). 
del Río Silala habían sido usadas por más de un siglo por Chile sin beneficio alguno para Bolivia, y anunció que pondría este asunto en la agenda bilateral ${ }^{18}$.

21. Poco después, el 31 de mayo de 1996, el Embajador de Bolivia Teodosio Imaña Castro, Presidente de la Comisión Nacional de Soberanía y Límites del Ministerio de Relaciones Exteriores de Bolivia, confirmó que el Río Silala fluye de manera natural desde Bolivia hacia Chile, siguiendo un curso constante cerro abajo ${ }^{19}$.

22. Los documentos mencionados demuestran que desde fines del siglo XIX hasta al menos 1996, Bolivia consideraba que el Silala era un curso de agua internacional. Recién en 1997 comenzó a cambiar su postura a favor de una pretensión que el Silala es una vertiente cuyas aguas están ubicadas exclusivamente en territorio boliviano y por cuyo uso Chile debía pagar.

23. En efecto, el 14 de mayo de 1997, Bolivia resolvió "revocar y anular" la concesión otorgada a FCAB en 1908 para el uso de las aguas, afirmando que había desaparecido su objeto y finalidad debido al retiro de las locomotoras de vapor y a la inexistencia jurídica de la compañía. En esta Resolución Administrativa, Bolivia se refiere a las aguas Silala como "vertientes" y evita referirse al Silala como un río ${ }^{20}$.

24. Durante el 1999, Chile envió varias notas diplomáticas a Bolivia en las que expresaba su preocupación respecto a que las declaraciones hechas por autoridades bolivianas referidas a las aguas del Silala, como también un llamado a licitación para el uso de dichas aguas, no tomaban en consideración el carácter internacional del sistema del Río Silala y los derechos de Chile sobre su uso ${ }^{21}$. En respuesta, Bolivia aseguró que las aguas eran de carácter exclusivamente boliviano y negó que el Silala fuese un curso de agua internacional ${ }^{22}$.

25. El 25 de abril de 2000, Bolivia otorgó el uso de las aguas del Silala a la compañía privada boliviana DUCTEC S.R.L. por un plazo de cuarenta años. La concesión autorizaba la comercialización o exportación de las aguas para uso industrial y consumo humano,

18 Comunicado de Prensa del Ministerio de Relaciones Exteriores de Bolivia, 7 de mayo de 1996, en: El Diario, La PazBolivia (Anexo 14).

19 Entrevista con el Embajador de Bolivia, Teodosio Imaña Castro, 31 de mayo de 1996, en: Presencia, La Paz-Bolivia (Anexo 15).

20 Resolución Administrativa N 71/97 por la Prefectura del Departamento de Potosí-Bolivia, 14 de mayo de 1997 (Anexo 16).

21 Nota $N^{\circ}$ 474/71 del Consulado General de Chile en La Paz-Bolivia al Ministerio de Relaciones Exteriores y Culto de la República de Bolivia, 20 de mayo de 1999 (Anexo 17); Nota Nº17550 del Ministerio de Relaciones Exteriores de la República de Chile al Ministerio de Relaciones Exteriores y Culto de la República de Bolivia, 15 de septiembre de 1999 (Anexo 18); Nota No 1084/151 del Consulado General de Chile en La Paz-Bolivia al Ministerio de Relaciones Exteriores y Culto de la República de Bolivia, 14 de octubre de 1999 (Anexo 19); Nota Nº 022314 del Ministerio de Relaciones Exteriores de la República de Chile al Ministerio de Relaciones Exteriores y Culto de la República de Bolivia, 3 de diciembre de 1999 (Anexo 20).

22 Nota Nº GMI-656/99 del Ministerio de Relaciones Exteriores y Culto de la República de Bolivia al Consulado General de Chile, 3 de septiembre de 1999 (Anexo 21); Nota N GMI-815/99 del Ministerio de Relaciones Exteriores y Culto de la República de Bolivia al Ministerio de Relaciones Exteriores de la República de Chile, 16 de noviembre de 1999 (Anexo 22). 
presumiblemente a Chile, ya que la concesión excluía explícitamente su uso para servicios de agua potable y alcantarillado en Bolivia sin una concesión adicional de servicio público, como también para actividades mineras a favor de terceros en territorio boliviano $^{23}$. En mayo de 2000, DUCTEC intentó facturar a CODELCO y FCAB por su uso de las aguas del Silala, ignorando los derechos existentes de ambas compañías sobre el uso de dichas aguas en territorio chileno.

26. Chile objetó formalmente la concesión de las aguas a DUCTEC debido a que desconocía el carácter internacional del Río Silala y el derecho de Chile sobre el uso de sus aguas ${ }^{24}$.

27. Después de este intercambio de notas diplomáticas, Bolivia y Chile establecieron una comisión técnica y acordaron cooperar en la compilación de información acerca del área del Silala y en la elaboración de cartografía. Dichos esfuerzos conjuntos incluyó un vuelo aerofotogramético en noviembre de 2001.

28. El 26 de febrero de 2002, el Ministerio de Relaciones Exteriores de Bolivia negó públicamente que hubiera algún tipo de negociación bilateral con relación a las aguas del Silala. Afirmó que las aguas del Silala no fluían de manera natural hacia Chile y que pertenecían exclusivamente a Bolivia. También anunció como posibles cursos de acción, cerrar el flujo de las aguas hacia Chile o recurrir a procedimientos internacionales ante un tribunal ad hoc tribunal o ante esta Corte ${ }^{25}$.

29. Chile rechazó que Bolivia calificara al Río Silala como un curso de agua exclusivamente boliviano. Asimismo expresó su rechazo hacia cualquier medida que pudiese entorpecer el flujo de las aguas del Río Silala hacia el interior del territorio chileno ${ }^{26}$.

30. En 2004, Bolivia y Chile crearon un Grupo de Trabajo cuyo propósito era continuar analizando el asunto del Silala y proporcionar elementos para un entendimiento común respecto del curso de agua. En 2006, el asunto del Silala también fue incluido como uno de los puntos en una agenda bilateral establecida entre los dos Estados.

31. No hubo avances hasta el 2008 cuando Bolivia aceptó retomar el trabajo técnico conjunto como base para un posible acuerdo preliminar. Se sostuvieron reuniones hasta el 2009, en que el Grupo de Trabajo logró algunos avances hacia definir un régimen de utilización y protección de las aguas del Silala, incluyendo la realización de estudios técnicos conjuntos del sistema hidrológico del Silala.

23 Contrato de Concesión de Uso y Aprovechamiento de los Manantiales del Silala entre el Superintendente de Saneamiento Básico de Bolivia y DUCTEC S.R.L., 25 de abril de 2000 (Anexo 23).

24 Nota N 006738 del Ministerio de Relaciones Exteriores de la República de Chile al Ministerio de Relaciones Exteriores y Culto de la República de Bolivia, 27 de abril de 2000 (Anexo 24).

25 Comunicación $N^{\circ} 143$ del Consulado General de Chile en Bolivia adjuntando un Comunicado de Prensa del Ministerio de Relaciones Exteriores de Bolivia, 26 de febrero de 2002 (Anexo 25).

26 Comunicado de Prensa del Ministerio de Relaciones Exteriores de Chile, 4 de marzo de 2002 (Anexo 26). 
32. En julio de 2010, en una reunión acerca de los puntos de la agenda bilateral, Bolivia volvió a su postura original de ser propietario del 100\% de las aguas del Silala e introdujo la noción de que Chile debía pagar una compensación por su centenario uso de las aguas como parte de cualquier acuerdo relacionado con el Silala (caracterizada por Bolivia como la "deuda histórica" de Chile). Dicha posición es directamente opuesta a la caracterización de larga data que mantuvo Bolivia del Silala como un río internacional. La misma no se conforma al derecho internacional consuetudinario y resulta inaceptable para Chile.

33. En octubre de 2010, el Grupo de Trabajo se reunió una vez más. En esta ocasión, Bolivia insistió en su propuesta de incorporar la "deuda histórica" de Chile como parte de un régimen de utilización de las aguas del Silala, lo cual fue rechazado nuevamente por Chile. La intransigencia de Bolivia en este asunto hizo que fuera infructuoso realizar estudios técnicos conjuntos del sistema hidrológico y las reuniones del Grupo de Trabajo se dieron por terminadas sin resultado alguno.

34. El 7 de mayo de 2012, para asegurarse que se preservaran sus derechos como Estado ribereño sobre la utilización de las aguas del Silala, Chile solicitó información sobre varios proyectos en el área del Silala que habían sido anunciados por el Gobernador del Departamento de Potosí, incluyendo la construcción de una planta piscícola, una represa y una planta embotelladora de agua mineral ${ }^{27}$. Bolivia no respondió a la solicitud de Chile.

35. Chile reiteró su solicitud de información el 9 de octubre de $2012^{28}$. El 25 de octubre de 2012, Bolivia respondió negando una vez más que el Silala fuera un río internacional y reafirmando sus derechos plenos y soberanos sobre el uso y aprovechamiento de sus aguas $^{29}$. A continuación hubo un intercambio de notas diplomáticas, en las que Bolivia mantuvo su postura.

36. En la medida de lo que Chile ha podido determinar, Bolivia ha construido un estanque piscícola, un puesto militar y casas al lado del Río Silala. Dichos proyectos podrían tener efectos negativos en la calidad y cantidad de las aguas del Río Silala. Sin embargo Chile no ha sido informado ni de planes para tales medidas ni de alguna medida que Bolivia haya tomado para evitar y controlar cualquier contaminación resultante en las aguas del Río Silala.

27 Nota $N^{\circ} 199 / 39$ del Consulado General de Chile in La Paz-Bolivia al Ministerio de Relaciones Exteriores del Estado Plurinacional de Bolivia, 7 de mayo de 2012 (Anexo 27).

28 Nota No 389/149 del Consulado General de Chile in La Paz-Bolivia al Ministerio de Relaciones Exteriores del Estado Plurinacional de Bolivia, 9 de octubre de 2012 (Anexo 28).

29 Nota N ${ }^{\circ}$ VRE-DGRB-UAM-020663/2012 del Ministerio de Relaciones Exteriores del Estado Plurinacional de Bolivia al Consulado General de Chile, 25 de octubre de 2012 (Anexo 29). 
37. El 23 de marzo de 2016, con motivo de la celebración anual en Bolivia del Día del Mar, el Presidente del país Evo Morales anunció que Bolivia tenía la intención de defender las aguas del Silala ante las instancias internacionales competentes. El Presidente Morales afirmó que: "Cada día, Chile se aprovecha ilegal y arteramente de ese recurso natural sin compensar ni un centavo. Este acto abusivo y arbitrario, que vulnera nuestro patrimonio, no puede continuar." 30 Dos días después, el Presidente Morales acusó a Chile de estar "robando agua del departamento de Potosí" y anunció la decisión de Bolivia de presentar una demanda ante la Corte Internacional de Justicia ${ }^{31}$.

38. El 29 de marzo de 2016, el Presidente Morales hizo una visita a terreno al Río Silala, junto con el Canciller David Choquehuanca, el Vice-canciller Juan Carlos Alurralde y el Procurador general del Estado Héctor Arce, entre otras altas autoridades. En esta ocasión, el Presidente Morales dejó en claro que "las autoridades chilenas mienten al afirmar que este [el Silala] es un río internacional” y de nuevo afirmó que sus aguas son exclusivamente de Bolivia ${ }^{32}$.

39. Así, Bolivia sigue negando y limitando los derechos de Chile como país ribereño al uso de cursos de agua internacionales compartidos por ambos países (tal como lo ha hecho con un segundo río, el Río Lauca, cuyas aguas son parcialmente usadas por Chile con fines de riego ${ }^{33}$ ).

40. Las comunicaciones entre Chile y Bolivia desde 1999 con relación a las aguas del Silala y, en particular, las recientes acciones y declaraciones realizadas por Bolivia demuestran que existe una controversia entre ambos Estados relativa al carácter jurídico del Río Silala como un curso de agua internacional y el derecho de Chile a la utilización de sus aguas de conformidad con el derecho internacional consuetudinario. En cuanto a esta controversia, ambos Estados mantienen puntos de vista opuestos e irreconciliables.

41. En estas circunstancias, Chile ha resuelto solicitar a la Corte su decisión sobre su disputa jurídica con Bolivia respecto al carácter del sistema del Río Silala como un curso de agua internacional y los derechos de Chile al ser un país ribereño.

30 Prensa Palacio, "Presidente Morales instruye estudiar alternativas jurídicas para defender aguas del Silala", La Paz-Bolivia, 23 Marzo 2016, disponible en: http://www.presidencia.gob.bo/fuente/noticia.php?cod=4185 (Anexo 6.1). Véase también La Nación, "Evo Morales anuncia que acudirá a instancias internacionales por aguas del Silala", La Paz-Bolivia, 23 de marzo de 2016, disponible en: http://www.lanacion.cl/noticias/mundo/bolivia/evo-morales-anuncia-que-acudira-a-instanciasinternacionales-por-aguas/2016-03-23/113759.html (Anexo 6.2).

31 Página Siete Digital, "Bolivia demandará a Chile por el Silala en La Haya", La Paz - Bolivia, 26 de marzo de 2016, disponible en: http://www.paginasiete.bo/nacional/2016/3/26/bolivia-demandara-chile-silala-haya-91113.html (Anexo 6.3).

32 Camiri.net, "Evo muestra al mundo que aguas del Silala son de Bolivia", 29 de marzo de 2016, disponible en: http://www.camiri.net/?p=22817 (Anexo 6.4).

33 El asunto concerniente al Río Lauca surgió por primera vez a finales de la década de 1930. Se centró en el uso que hacía Chile de sus aguas para riego en el Valle de Azapa que finalmente llevó a un quiebre en las relaciones diplomáticas entre Bolivia y Chile en 1962. 


\section{Fundamentos de derecho}

42. El uso de cursos de agua internacionales se rige por el derecho internacional consuetudinario. Los principios del derecho internacional consuetudinario sobre los usos de los cursos de agua con fines distintos de la navegación quedan demostrados por la Convención sobre el derecho de los usos de los cursos de agua internacionales para fines distintos de la navegación (en adelante, "UNWC”, por su sigla en inglés), suscrito en Nueva York el 21 de mayo de 1997 y que entró en vigor el 17 de agosto de $2014^{34}$, por la jurisprudencia de esta Corte y otras cortes y tribunales, y por la práctica de los Estados.

\section{La definición de un curso de agua internacional según el derecho internacional consuetudinario}

43. Según el derecho internacional general, un curso de agua que atraviesa dos o más Estados es considerado como un "curso de agua internacional". El Artículo 2 de la UNWC refleja el derecho internacional consuetudinario, estableciendo que:

“(a) Por "curso de agua" se entenderá un sistema de aguas de superficie y subterráneas que, en virtud de su relación física, constituyen un conjunto unitario y normalmente fluyen a una desembocadura común;

(b) Por "curso de agua internacional" se entenderá un curso de agua alguna de cuyas partes se encuentran en Estados distintos";

44. No cabe duda de que el Río Silala es un curso de agua internacional de acuerdo al derecho internacional consuetudinario. La cuenca del Río Silala presenta una pendiente ininterrumpida y regular de aproximadamente $4,3 \%$ en promedio, desde sus orígenes en Bolivia hasta que llega al Río Inacaliri de Chile. Sus cabeceras están las Quebradas bolivianas de Cajones y Orientales, a unos 4.360 y 4.421 metros sobre el nivel del mar, respectivamente. El Río Silala atraviesa el límite internacional e ingresa a territorio chileno a unos 4.278 metros sobre el nivel del mar. En varios tramos en Bolivia y en Chile, sus aguas fluyen por quebradas que fueron labradas en el transcurso de miles de años, una evidencia clara de que el Silala es un río y no producto de una canalización reciente.

45. Durante al menos 93 años Bolivia ha reconocido consistentemente el carácter de curso de agua internacional del Silala. Ha aceptado la representación cartográfica del Silala

\footnotetext{
34 Convención sobre el derecho de los usos de los cursos de agua internacionales para fines distintos de la navegación, Nueva York, 21 de mayo de 1997, Documento ONU A/RES/51/229 (1997), disponible en: https://treaties.un.org/doc/Treaties/1998/09/19980925\%2006-30\%20PM/Ch_XXVII_12p.pdf (Anexo 30).
} 
como río en numerosas ocasiones, incluyendo el Mapa oficial firmado y anexado al Tratado de Paz y Amistad de 1904. Bolivia no puede negar ahora el hecho que el Silala es un río internacional al referirse a él como "manantiales" o "vertientes". Además, el hecho que las aguas que forman el Río Silala emerjan a la superficie a través de una vertiente no evita que esas aguas formen un curso de agua internacional.

\section{El principio de utilización equitativa y razonable}

46. El principio de utilización equitativa y razonable de cursos de agua internacionales es derecho internacional consuetudinario. Tal como la Corte ha admitido, este principio se basa en la comunidad de intereses de todos los Estados ribereños en el uso de un curso de agua compartido ${ }^{35}$.

47. Chile sostiene que la aplicación del derecho internacional consuetudinario sobre los usos de los cursos de agua internacionales para fines distintos de la navegación confirma el uso pasado y presente de Chile de las aguas del Río Silala, cuyo flujo atraviesa el límite internacional, como equitativo y razonable.

3. Otras obligaciones de Bolivia según el derecho internacional consuetudinario

48. De acuerdo con el derecho internacional consuetudinario, Bolivia tiene la obligación de cooperar y evitar daños transfronterizos al uso de las aguas del Río Silala en Chile. Bolivia debe además notificar e informar a Chile sobre medidas proyectadas que puedan generar efectos perjudiciales sobre la utilización chilena de esas aguas. Dicha notificación debe ir acompañada por información y datos técnicos disponibles, incluyendo los resultados de cualquier evaluación de impacto ambiental, para permitir a Chile evaluar sus posibles efectos.

49. Bolivia ha violado dichas obligaciones al rehusar atender la solicitud de Chile del año 2012 por información acerca de la construcción de un estanque piscícola y otros proyectos. Además, tampoco informó a Chile sobre la construcción de un puesto militar y viviendas que podrían tener un impacto perjudicial en el Río Silala que afectara negativamente a Chile. Tampoco hay pruebas que Bolivia haya tomado en consideración el impacto ambiental de dichas instalaciones y su efecto potencial en las aguas del Río Silala que fluyen hacia Chile.

35 Proyecto Gabčíkovo-Nagymaros (Hungria/Eslovaquia), Fallo, Informes de la CIJ 1997, p. 7, en p. 56, párr. 85. 


\section{Decisión solicitada}

50. Con base en la exposición de hecho y derecho precedente, y reservándose el derecho a modificar las siguientes peticiones, Chile solicita a la Corte que juzgue y declare que:

(a) El sistema del Río Silala, junto con las porciones subterráneas de su sistema, es un curso de agua internacional, cuyo uso se rige por el derecho internacional consuetudinario;

(b) Chile tiene derecho al uso equitativo y razonable de las aguas del sistema del Río Silala de conformidad con el derecho internacional consuetudinario;

(c) Bajo el estándar de utilización equitativa y razonable, Chile tiene derecho al uso que actualmente hace de las aguas del Río Silala;

(d) Bolivia tiene la obligación de tomar todas las medidas apropiadas para prevenir y controlar la contaminación y otras formas de daño a Chile que resulten de sus actividades en las cercanías del Río Silala;

(e) Bolivia tiene la obligación de cooperar y de proporcionar a Chile una notificación oportuna de medidas planificadas que pudiesen tener un efecto negativo sobre recursos hídricos compartidos, de intercambiar datos e información y de realizar, cuando proceda, una evaluación de impacto ambiental, para permitir que Chile pueda evaluar los posibles efectos de tales medidas planificadas, obligaciones que Bolivia ha incumplido.

51. Chile se reserva el derecho a complementar, modificar o ampliar la presente Solicitud en el transcurso del procedimiento.

52. Chile se reserva asimismo el derecho a solicitar que la Corte indique medidas cautelares, en caso que Bolivia incurriera en cualquier conducta que pudiera tener un efecto negativo en el actual uso que hace Chile de las aguas del Río Silala.

53. Chile ejercerá el derecho otorgado por el Artículo 31 del Estatuto de la Corte para designar a una persona que actúe como Juez ad hoc. Informará a la Corte de su decisión oportunamente.

54. La abajo firmante ha sido designada por el Gobierno de Chile para actuar como Agente para los fines de esto procedimiento. Se solicita que toda comunicación relativa al 
Revista Tribuna Internacional

Volumen $5 \cdot \mathrm{N}^{\circ}$ 9 $・ 2016 \bullet$ pp. 199-211

ISSN 0719-482X (versión en línea)

presente caso sea enviada a la Embajada de la República de Chile en los Países Bajos, Mauritskade 51, 2514 HG, La Haya, Los Países Bajos.

Presentada respetuosamente,

La Haya, 6 de junio, 2016.

Ximena Fuentes T.

Agente de la República de Chile 